\title{
Adenovirus Release from the Infected Cell as a Key Factor for Adenovirus Oncolysis
}

\author{
Alena Gros ${ }^{1, *}$ and Sonia Guedan ${ }^{2}$ \\ ${ }^{1}$ Currently at Center for Cancer Research, National Cancer Institute, US National Institutes of Health, Bethesda, \\ Maryland, USA \\ ${ }^{2}$ Institut Català d'Oncologia (ICO), Av Gran Via s/n Km 2,7, L'Hospitalet de Llobregat, 08907 Barcelona, Spain
}

\begin{abstract}
Adenovirus release is not triggered until late times after viral infection, when the adenovirus death protein (ADP) accumulates to induce viral egress. Thus, the natural rate of adenovirus release may hinder the spread of oncolytic adenoviruses. Several experimental approaches have provided evidence indicating that promoting adenovirus release can be used to enhance their therapeutic potential. This review briefly summarizes what is known about the mechanism of adenovirus release and describes three different strategies, ADP overexpression, apoptosis induction, and bioselection, which can be used to enhance adenovirus release. Finally we will discuss some of the future perspectives that will contribute to the better use of progeny release for the improvement of the antitumor activity of oncolytic adenoviruses.
\end{abstract}

Keywords: Adenovirus, progeny release, cell-to-cell spread, oncolytic potency.

\section{INTRODUCTION}

Viral spread and pathogenesis is determined by the efficiency of viral exit from the infected cell. The release of adenovirus is rather inefficient since it occurs long after infection when the adenovirus death protein (ADP) has accumulated to promote viral egress. Only $20 \%$ of the adenovirus produced is released by $48 \mathrm{~h}$ post-infection. This has led to the idea that the natural rate of adenovirus release hinders the intratumoral spread of oncolytic adenoviruses. The large plaque phenotype indicative of improved cell-tocell spread yielded with specific genetic modifications of adenovirus was the first evidence that augmenting progeny release could be used to enhance the spread of oncolytic adenoviruses. Overexpression of ADP [1] or functional deletion of E1b-19kD [2] were able to enhance the cytotoxicity of adenovirus by inducing early cell lysis and enhancing progeny release. Moreover, Wein and collaborators used a mathematical model to study the complex interactions between tumor cell growth, virus replication, and the immune system. They concluded that viral replication would only eliminate the tumor when the rate of cell lysis and progeny release outpaced tumor cell growth [3]. In contrast, another study by Wodarz suggested that maximizing the death rate of infected tumor cells could decrease viral production and result in suboptimal outcome of treatment [4]. Supporting Wein's hypothesis, several reports have demonstrated that genetic modifications which improve adenovirus release enhance the antitumor activity of oncolytic adenoviruses in tumor xenografts $[5,6]$. In addition, both in vitro and in vivo bioselection of mutants with improved spread or oncolytic potency led to releaseenhancing mutations, highlighting the importance of adenovirus release for oncolysis [7-9]. According to this

\footnotetext{
*Addressed Correspondence to this Author at the Center for Cancer Research, National Cancer Institute, US National Institutes of Health, Bethesda, Maryland, USA; Tel: (301)4022397; Fax: (301)4021738; E-mail: alena.gros@nih.gov
}

evidence, the rate of progeny release is a key parameter controlling the spread and antitumoral efficacy of oncolytic adenoviruses. This review outlines the biology of adenovirus cell death and progeny release, summarizes the strategies which can be used to enhance the release of oncolytic adenoviruses, and highlights several considerations to choose a release enhancing modification.

\section{BIOLOGY OF ADENOVIRUS CELL DEATH AND PROGENY RELEASE}

To date, the mechanism of adenovirus cell lysis and progeny release remains poorly understood. Several different groups have attempted to characterize adenovirus-induced cell death at late stages of infection, but the exact mechanism remains controversial. Zhang and Schneider first demonstrated that host cell shutoff of protein synthesis was necessary to facilitate cell lysis and release of infectious progeny virus particles [10]. Treatment of adenovirusinfected cells with 2-aminopurine prevented host-cell shutoff and yielded a 200-fold reduction of progeny release without significantly affecting viral production. However, the contribution of this process to adenovirus release remains to be elucidated. Subsequent studies revealed that adenovirus encodes for several proteins which both inhibit or promote cell death and act in concert to control the fate of the infected cell and the exit of the viral progeny. E1B-19kD together with E1B-55kD and E4orf6 are expressed early after infection and act as antiapoptotic proteins preventing premature apoptotic cell death induced by E1A [11-13]. Deletion or loss of function of E1b-19kD yields a large plaque phenotype, indicative of improved cell-to-cell spread due to enhanced cell killing and progeny release, but has also been reported to decrease viral production $[8,14]$. Therefore, the importance of this protein relies on its ability to delay cell death to ensure efficient viral replication and generation of new virions.

At late times after adenovirus infection, virions accumulate in the nucleus, intermediate filaments are disrupted, 2010 Bentham Open 
the cell rounds-up evidencing the typical cytophathic effect (CPE), and cell membrane permeabilization allows virions to escape into the extracellular medium. The E3-11.6K protein, also known as adenovirus death protein (ADP), has a major role in this process. Deletion of this protein results in an impaired release without affecting viral production, and cells infected with these mutants have small, slow developing plaques. Although the exact function of this protein is still unknown, the accumulation of this protein late in the adenovirus cycle is required for efficient cell lysis and release of viral particles into the extracellular medium [15, 16]. Interestingly, although E3-11.6K is encoded within the early E3 transcriptional unit it is synthesized only in small amounts from the E3 promoter, but very abundantly from the major late promoter starting at about 20 to $25 \mathrm{~h}$ postinfection [17]. Additionally to ADP, E4orf4 has also been found to induce cell death [18] and has been suggested to collaborate with ADP inducing cell lysis and progeny release. In fact, although less evident than ADP-deleted viruses, E4orf4-null viruses exhibit a prolonged delay of cell death [19], supporting a role for E4orf4 in this process.

More recently, a work from Abou el Hassan et al. indicated that the cell killing induced by an oncolytic adenovirus was independent from the basic apoptotic machinery and termed the oncolysis induced by the virus as 'necrosis-like' [20]. In a later study, Ito et al. coupled, for the first time, adenovirus induced cell death with autophagy. They found that infection of glioma cells with a replicative adenovirus with E1A expression controlled under the hTERT promoter (hTERT-Ad) induced acidic vesicular organelle development and increased the localization of LC3 to the autophagosome membrane (enhanced LC3-II to LC3-I ratio), two typical features of autophagy [21]. Supporting this type of cell death, Jiang et al. also described Ad $\Delta 24 R G D$ induced autophagic cell death in glioma stem cells, as indicated by accumulation of Atg5 and LC3-II protein and autophagic vacuoles [22]. Furthermore, enhancement of autophagy in glioma cells through combination with RAD001 improved the antitumoral effect of oncolytic adenovirus Ad $\triangle 24$ RGD in vitro and in vivo [23]. Contrary to these results, Baird et al. found that 3-methyladenine and chloroquine, two drugs which inhibit early autophagic signaling and autophagosome and lysosome fusion, respectively, augmented virus-induced death in ovarian cancer cell lines in a dose dependent manner [24]. A more recent work by Ulasov et al. carefully studied apoptosis and autophagy involvement in adenovirus induced oncolysis in glioma cells. Their results, which include both non-classical apoptosis and autophagy features, indicated that apoptosis may not be a terminal stage of virus-induced toxicity and that autophagy could explain some of the proapoptotic features observed [25]. All together, these results discard classical apoptosis and provide evidence for autophagy induction at late stages of adenovirus infection. However, further experiments will be required to demonstrate whether autophagy contributes to adenovirus induced cell death or acts as a cell survival response to adenovirus infection.

\section{METHODS TO INCREASE ADENOVIRAL SPREAD}

Several encouraging strategies are being explored to generate oncolytic adenoviruses with enhanced release and improved potency. In this review, the various viruses will be organized into three main categories, according to the strategy that has been used to enhance their release. The first category includes oncolytic adenoviruses which overexpress the E3 protein ADP. The second category includes strategies to induce apoptosis in the infected cell. Finally, the third category includes those mutants obtained through bioselection of randomly mutagenized pools of adenoviruses. Table $\mathbf{1}$ summarizes current replication-competent adenoviruses with enhanced release, and their main characteristics.

\section{ADP Overexpression}

After the identification of ADP as a key element for efficient cell lysis and release of virions from infected cells, a number of adenoviruses that overexpress ADP have been constructed with the aim of improving their oncolytic potential. In these viruses, ADP overexpression has been achieved by using two main strategies.

The first strategy consists of shifting the expression of ADP from late to early to accumulate larger amounts of ADP at earlier stages and make cell lysis and release more efficient. To accomplish this, Doronin et al. deleted all the E3 genes except ADP or all the E3 genes except E3-12.5K and ADP, to generate KD1 and KD3, respectively [1]. The deletion of these genes changes the fine transcriptional balance of the E3 transcriptional unit favoring the transcript that encodes ADP leading to its overexpression. KD1 and KD3 also have two E1A mutations that preclude binding of E1A proteins to $\mathrm{p} 300$ and $\mathrm{pRB}$, and render these viruses selective for dividing cancer cells. Using these viruses, they demonstrated for the first time that ADP overexpression produces early cytophatic effect and increases cell-to-cell spread without impairing viral production due to a premature cell lysis. Importantly, both KD1 and KD3 were more effective at suppressing subcutaneous lung cancer tumors in vivo than a control virus, which expressed wild-type levels of ADP. Later, this strategy has been used by the same authors for the construction of two replication competent adenoviruses expressing a wild-type E1A protein, VRX-006 (lacking all the E3 genes except 11.6K) and VRX-007 (lacking all the E3 genes but $12.5 \mathrm{~K}$ and $11.6 \mathrm{~K}$ ) and several other oncolytic adenoviruses such as VRX-009 and VRX011 (based on VRX-007) or KD1-SPB (based on KD1). In each case, it was found that ADP overexpression produced early cytophatic effect, early cell lysis, large plaque, and increased cell-to-cell spread resulting in an improved therapeutic potential $[5,6,26,27]$.

The second strategy involves the replacement of the entire E3 coding region with an expression cassette containing the $A D P$ gene controlled by a promoter. Using this approach, Ramachandra et al. constructed the oncolytic adenovirus 01/PEME, in which an extra copy of the viral MLP was placed into the E3 region for overexpression of ADP [28]. 01/PEME displayed increased CPE in cell culture and enhanced antitumor activity in vivo compared to viruses that do not overexpress ADP. In another study, ADP was expressed under the control of the CMV promoter (YKLcADP) [29]. ADP expression from the CMV promoter produced earlier cell lysis and enhanced viral release compared to ADP expression from the extra MLP (YKL- 
Table 1. Examples of Replication-Competent Adenoviruses with Enhanced Progeny Release

\begin{tabular}{|c|c|c|c|c|c|}
\hline Viruses & Genetic Modifications to Increase Release & ADP & E3 Genes Deleted & $\begin{array}{l}\text { Selective } \\
\text { Replication }\end{array}$ & Reference \\
\hline \multicolumn{6}{|c|}{ ADP overexpression } \\
\hline $\begin{array}{l}\text { KD1 } \\
\text { KD3 }\end{array}$ & deletion of E3 genes & overexpressed & $\begin{array}{l}\text { all but } 11.6 \mathrm{~K} \\
\text { all but } 12.5 \text { and } 11.6 \mathrm{~K}\end{array}$ & YES & {$[1]$} \\
\hline $\begin{array}{l}\text { VRX-006 } \\
\text { VRX-007 }\end{array}$ & deletion of E3 genes & overexpressed & $\begin{array}{l}\text { all but } 11.6 \mathrm{~K} \\
\text { all but } 12.5 \mathrm{~K} \text { and } 11.6 \mathrm{~K}\end{array}$ & NO & {$[27]$} \\
\hline 01/PEME & Insertion of a cassette containing $a d p$ gene and MLP & overexpressed & all but $12.5 \mathrm{~K}$ & YES & [28] \\
\hline $\begin{array}{l}\text { YKL-cADP } \\
\text { YKL-mADP }\end{array}$ & $\begin{array}{c}\text { Insertion of a cassette containing } a d p \text { gene and: } \\
\text { CMV promoter } \\
\text { MLP }\end{array}$ & overexpressed & all but $12.5 \mathrm{~K}$ & YES & {$[29]$} \\
\hline $\begin{array}{c}\text { Ad5-yCD/ } \\
\text { mutTKSR39-rep-ADP }\end{array}$ & $\begin{array}{l}\text { Insertion of a cassette containing } a d p \text { gene and: } \\
\text { CMV promoter }\end{array}$ & overexpressed & all & YES & [30-31] \\
\hline d1716 & C-terminal truncation of ADP protein & overexpressed & none & NO & {$[34,36]$} \\
\hline d1732 & $\begin{array}{l}\text { Mutation of the splicing acceptor } \\
\text { near } a d p \text { coding sequence }\end{array}$ & overexpressed & none & NO & {$[35,36]$} \\
\hline \multicolumn{6}{|c|}{ Apoptosis induction } \\
\hline Ad337 & E1b-19kD deletion & wild-type & $\begin{array}{c}\text { E3B }(14.7 \mathrm{~K} \\
14.5 \mathrm{~K}, 10.4 \mathrm{~K})\end{array}$ & YES & {$[2,37]$} \\
\hline $\mathrm{d} 1250$ & E1b-19kD deletion & wild-type & none & YES & {$[38,39]$} \\
\hline Adp53rc & Insertion of p53 gene after the fiber gene & deleted & all & NO & {$[50]$} \\
\hline $\mathrm{Ad} \Delta 24-\mathrm{p} 53$ & $\begin{array}{l}\text { Insertion of p53 expression cassette } \\
\text { in the deleted E3 region }\end{array}$ & deleted & all & YES & {$[51-53]$} \\
\hline Ad5/35.1R-E1A/TRAIL & $\begin{array}{l}\text { Insertion of E1A-TRAIL expression cassette } \\
\text { replacing the deleted E1 region }\end{array}$ & wild-type & none & YES & {$[57,58]$} \\
\hline ZD55-TRAIL & $\begin{array}{l}\text { Insertion of CMV-TRAIL cassette replacing the } \\
\text { deleted E1b-55K gene }\end{array}$ & wild-type & none & YES & {$[59,60]$} \\
\hline \multicolumn{6}{|c|}{ Bioselection of mutants } \\
\hline Lp53 & C-terminal truncation of i-leader protein & deleted & all but $12.5 \mathrm{~K}$ & NO & {$[8]$} \\
\hline $\begin{array}{l}\text { Lp11 } \\
\text { Lp26 }\end{array}$ & $\begin{array}{c}\text { Defective in E1b-19K } \\
\text { Defective in E1b-19K and E1b-55K }\end{array}$ & deleted & all but $12.5 \mathrm{~K}$ & NO & {$[8]$} \\
\hline $\begin{array}{l}\text { ONYX-201 } \\
\text { ONYX-203 }\end{array}$ & C-terminal truncation of i-leader protein & wild-type & none & NO & [9] \\
\hline AdT1 & C-terminal truncation of E3-19kD protein & wild-type & none & NO & [7] \\
\hline
\end{tabular}

mADP). Moreover, the enhanced potency of YKL-cADP adenovirus over YKL-mADP also translated into a greater antitumor efficacy and survival advantage in vivo in both a cervical and hepatoma xenograft tumor models. Finally, ADP expression under the control of the CMV promoter has also been used by another group to generate a secondgeneration armed adenovirus, Ad5-yCD/mutTKSR39repADP [30]. Importantly, the safety of this virus has been established in a clinical trial [31], and now its efficacy is being evaluated in a phase III randomized clinical trial.

It is important to note that all the strategies described above to overexpress ADP require partial or total deletion of the E3 region (Table 1). Although the loss of function of these proteins can be underestimated in vitro, several studies provide clear evidence demonstrating that deletion of some of these proteins favor the clearance of the virus in immunocompetent models [32, 33]. As an alternative to the previously mentioned viruses, $d l 716$ and $d l 732$ mutants overexpress ADP while preserving the E3 immunomodulatory functions. These two mutants were used in early virologic works to study the E3 complex transcription unit as a model to understand alternative pre-mRNA processing [34, 35]. However, it was not until 2003 that these viruses were described to display large-plaque phenotype [36]. The $d l 732$ mutant contains a 60 bp deletion which affects a splicing acceptor near the ADP coding sequence and results in earlier transcript synthesis and overexpression of this gene. The $d l 716$ mutant has a 22 bp deletion that induces a c-terminal truncation of the ADP protein. Although to date these genetic modifications have not been applied to enhance the release of an oncolytic adenovirus, the preservation of the E3 immunomodulatory functions could be beneficial in presence 
of an immune system, and therefore they deserve further investigation.

\section{Apoptosis Induction}

The first evidence that apoptosis could enhance progeny release of replicating adenoviruses was the large plaque phenotype displayed by an E1b-19K deleted adenovirus (d1337). Failure of this virus to inhibit E1A induced apoptosis during adenovirus infection led to premature cell death and enhanced release and spread [2]. Moreover, the d1337 mutant greatly enhanced the apoptotic effect of conventional chemotherapies, such as cisplatin and paclitaxel [2] in vitro, and was also better than wild-type adenovirus at reducing tumor growth in A549 tumor xenografts [37]. Soon after these earlier studies, another group further studied the selectivity and toxicity of adenoviral mutants lacking the E1b-19K gene with and without E3B gene expression. Overall, the adenoviral mutant with E1b-19K deletion and E3B retention (d1250) displayed enhanced viral spread and increased antitumoral potency compared to wild-type adenovirus in a variety of in vitro and in vivo tumor models $[38,39]$. In addition, this mutant showed tumor necrosis factor-enhanced cancer selectivity due to genetic blocks in apoptosis pathways in cancer cells [38, 39]. Importantly, in all these studies, E1b-19K deletion did not impair viral replication, and both d1337 and d1250 grew to wild type levels, and sometimes even more. However, it is important to note, that in earlier virologic studies, E1b-19K mutants were found to be defective in non-complementing cell lines [14].

Another work presented by Mi et al. also supports the finding that apoptosis induction can result in earlier release of virions from infected cells. In this work, they used a system in which expression of a mutated form of I- $k \mathrm{~B}$ (that avoids NF-kB activation) by an adenoviral vector (Ad.ikB $\mathrm{B}_{\mathrm{M}}$ ) sensitized tumor cells to recombinant human TNF- $\alpha-$ mediated apoptosis [40]. Although the repeated intratumoral administration of TNF- $\alpha$ in mice bearing tumors of HeLa cells infected with $A d . i k B_{M}$ had antitumor effect, this approach is not applicable in clinical settings because of potential side effects from systemically applied TNF and expression of $\mathrm{I}-k \mathrm{~B}_{\mathrm{M}}$ in non tumor tissues. However, this system served as a model to demonstrate that apoptosis induced after virion assembly facilitates virion release and enhances intratumoral spread. By contrast, apoptosis induced during viral DNA replication compromised virus production, indicating that careful timing of cell lysis is crucial. Importantly, this work demonstrated that apoptotic cell death leads to the engulfment of progeny virions into apoptotic bodies which can spread from cell to cell through phagocytosis of these apoptotic bodies. This cell death mode may be very valuable given the resistance of this type of spread to the presence of neutralizing antibodies.

A different strategy to induce apoptosis in infected cells is to arm viruses with exogenous transgenes that promote apoptosis. The p53 tumor suppressor protein is an attractive candidate that has been used for this purpose. p53 gene transfer has been shown to induce growth inhibition and apoptosis in a variety of cancer types [41-44]. By contrast, p53 gene transfer is relatively nontoxic to normal cells [45, 46]. Moreover, it has been suggested that functional p53 can promote the adenovirus lytic cycle [47-49]. On the basis of these observations, two independent groups exploited the use of p53 with the aim of improving oncolytic potency. Sauthoff et al. demonstrated that late expression of p53 from a replicating E3-deleted virus improves tumor cell killing and viral spread without impairing viral replication [50]. Interestingly, p53 was more cytotoxic than ADP in cancer cells, but less cytotoxic than ADP in normal fibroblasts, improving therefore the specificity of tumor cell killing. p53 gene has also been inserted in place of the deleted E3 region in $\operatorname{Ad} \Delta 24$, to create $\operatorname{Ad} \Delta 24-\mathrm{p} 53$ [51]. $\operatorname{Ad} \Delta 24-\mathrm{p} 53$ exhibited enhanced oncolytic potency compared with $\operatorname{Ad} \Delta 24$ in most cancer cell lines tested independent of their p53 status. Moreover, $\operatorname{Ad} \Delta 24-\mathrm{p} 53$ was more efficient at suppressing tumor growth when compared to $\operatorname{Ad} \Delta 24$ in glioma [52] and neuroblastoma [53] xenograft models.

Another transgene that has been used to increase adenovirus spread is the TNF-related apoptosis-inducing ligand (TRAIL). TRAIL is a transmembrane protein that is processed into a soluble molecule able to kill many types of tumor cells via an apoptotic cascade [54], while not causing any toxicity in vivo [55, 56]. In the earliest study, Sova et al. constructed a tumor-targeted oncolytic adenovirus which expressed TRAIL in a tumor-selective manner. This virus did not contain the antiapoptotic E1b-55kD and $19 \mathrm{kD}$ genes, which can potentially inhibit TRAIL-mediated apoptosis. TRAIL expression by this virus improved virus release and cytotoxicity in a variety of human tumor cell lines in vitro $[57,58]$. In vivo, the TRAIL-expressing virus showed an enhanced antitumor activity compared to its parental virus in a variety of tumor models, including intravenous administration in a liver metastasis model [57] and intratumoral administration in s.c. glioblastoma xenografts [58]. The ability of TRAIL to enhance viral release has been confirmed by another group that created an oncolytic adenovirus that expressed TRAIL (ZD55-TRAIL) by replacing the E1b$55 \mathrm{kD}$ gene with a CMV-TRAIL cassette [59]. Interestingly, ZD55-TRAIL has been used in combination with chemotherapeutic agents, such as 5-FU [59] and cisplatin [60], resulting in a greater antitumor activity than either treatment alone.

\section{Bioselection}

Another approach to generate viruses with enhanced release involves bioselection of randomly mutagenized adenovirus by repeated passaging under carefully controlled conditions. This genetic selection yields point mutations that are compatible with viral replication and has the potential to assign novel functions to viral genes. In the earliest work, Subramanian et al. used a bioselection strategy to identify Ad5 genes that play a role in viral spread [8]. In this study, an Ad5 mutant (d1327 lacking most of the E3 region) with small-plaque phenotype was randomly mutagenized by exposure to UV, and 27 mutants that were able to revert the reduced spread of the d1327 were selected. 23 out of 27 mutants contained mutations affecting the function of the E1B-19kD protein, which led the authors to hypothesize an opposing effect of E1B-19kD and ADP in adenovirus spread. Although E1b-19kD mutants formed large plaques in the absence of ADP, the presence of the E3 region in an E1b$19 \mathrm{kD}$ mutant did not significantly enhance plaque size or cytolysis, suggesting that ADP may antagonize the function 
of E1B-19kD. Among the other compensatory mutants that were isolated, lp53 showed the most pronounced effect. This virus contains a single base pair change in the adenoviral genome resulting in a C-terminal truncation of the i-leader protein. The lp53 mutation showed additive effect with the $\mathrm{E} 3$ region and with a deletion of the E1B-19kD region. In contrast, the i-leader null mutation did not appear to influence viral spread. Therefore, the authors suggested that the full-length i-leader protein may be in an inactive conformation and may be functionally activated by proteolysis of the C-terminal region under specific conditions. Interestingly, a previous genetic selection of Ad5 mutants with enhanced oncolytic activity identified two mutants (ONYX-201 and ONYX-203) that also contained a Cterminal truncation in the i-leader protein [9]. This mutation appeared to be essential for the increased cytotoxic activity of these mutants, confirming the relevance of such mutations. Although the exact role of the i-leader protein in adenovirus replication is not clear, the fact that this protein encoded by the major late transcription unit is expressed prior to viral DNA replication led the authors to postulate that early accumulation of the truncated i-leader protein could accelerate viral DNA replication through an unknown mechanism.

Finally, in an effort to select a mutant with enhanced antitumor activity, our group extended the bioselection approach by performing repeated passages of a pool of Ad5 random mutants in an in vivo murine model of human cancer [7]. By using this approach, we isolated AdT1, a new mutant with improved viral release and enhanced systemic antitumor activity in vivo. A truncating mutation in the endoplasmic reticulum retention domain of the E3-19K protein (445A), which relocates the protein to the plasma membrane, was found to be the responsible for the enhanced release phenotype. How the relocalization of E3-19K improves adenovirus release is not clear to date, however, our results suggest that the $\mathrm{E} 3-19 \mathrm{~K}(445 \mathrm{~A})$ mutation is able to disrupt intracellular $\mathrm{Ca}^{2+}$ homeostasis and create membrane lesions that allow enhanced virus release. Further studies to elucidate the role of the new identified mutations in viral release, as well as the incorporation of these mutations in oncolytic adenoviruses become of increasing relevance.

\section{FUTURE DIRECTIONS}

Three main considerations must be taken into account when choosing a release enhancing modification: (a) modifications should not compromise virus replication, (b) point mutations or deletions rather than insertion of transgenes should be preferentially used to save space, and (c) deletion of E3 immunomodulatory proteins may affect the ability of the virus to evade the immune response and, therefore, should be minimized.

The importance of the immune response to oncolytic adenoviruses is paramount as the outcome of the therapy will depend, in great measure, on the interaction of the virus with the immune system [61]. Besides the implications of the deletions of E3 immunomodulatory proteins to enhance progeny release, it is possible that the improvement of adenovirus release, itself, could affect the immune response to an oncolytic adenovirus. It is generally believed that a prolonged infectious cycle (inefficient viral release) in the case of a highly immunogenic virus such as adenovirus would allow the immune system to rapidly neutralize the infection without allowing virus to spread [62]. The faster the virus is released from the infected cell, the less chance the virus has of being killed in the cell by cytotoxic $\mathrm{T}$ lymphocytes, NK cells, and phagocytic cells and the greater chance it has to infect another cell [15]. Contrary to this hypothesis, the presence of more extracellular virus could possibly trigger a stronger innate response and, in turn, attract a more powerful adaptative antiviral response leading to a faster clearance of the virus. Despite this, the enhancement of adenovirus release has been reported to improve the spread and antitumoral potency of oncolytic adenoviruses in nude mice bearing human tumor xenografts models. However, the benefits of oncolytic adenoviruses with enhanced release have never been tested properly in presence of an immune system and the consequences of an improved release on the immunogenicity of an oncolytic adenovirus remain unexplored. So far, only two viruses with release enhancing modifications have been studied in Syrian hamsters, a model that is semipermissive to adenovirus replication: VRX-007 (all E3 gens deleted except 12.5K and ADP)[63] and AdT1 (Ad5 with E3/19K-445A mutation, which relocalizes E3-19K to the plasma membrane) [7]. In both cases, these viruses demonstrated the same antitumor efficacy as their wild-type counterparts. However, the release enhancing modifications of these viruses may also compromise other immune evading functions of adenovirus, making conclusions hard to extract. A more detailed study of the impact of release enhancing genetic modifications on the immune response to oncolytic adenoviruses would help predict if viruses with improved progeny release require combination with immune suppression when the objective is purely virocentric, or whether they could boost the immune response against the tumor when the final goal is immunocentric [61].

In addition to the immune response, tumor stroma may also play a prominent role in inhibiting viral spread and tumor cell killing of oncolytic adenoviruses. Solid tumors are characterized by small groups of tumor cells surrounded by large areas of tumor-associated fibroblasts and extracellular matrix (ECM). In addition, unfavorable pressure gradients within established tumors also inhibit diffusion of large molecules. Degradation of ECM with proteolytic enzymes, such as relaxin or hyaluronidase, has been shown to reduce interstitial fluid pressure, enhance adenoviral spread and improve viral potency in vivo [64-66]. Therefore, combining modifications to increase adenovirus release with strategies to disrupt intratumoral barriers may be important to maximize spread efficiency. Further studies on the use of promoters which allow the replication of oncolytic adenoviruses in tumor-associated fibroblasts as well as in tumor cells together with strategies to improve the spread within this cell type would also be of interest, as this cell population has been considered a major barrier for efficient virus spread.

Finally, a better understanding of the mechanism of viral release and the type of cell death mode triggered should promote the development of novel vectors with improved progeny release and contribute to enhance the antitumoral activity of oncolytic adenoviruses. In this sense, the study of 
progeny release induced by other naked viruses could also produce new hypotheses. The process leading to viral egress of poliovirus or coxsackievirus, for example, has been extensively studied. Both viruses encode for small highly hydrophobic proteins, capable of modifying the infected cells permeability to ions or small molecules, also known as viroporins [67-69]. A protein with the ability to modify permeability has not yet been described for adenovirus, although several hypotheses suggest that the adenovirus death protein (ADP) could induce cell lysis and release through a similar mechanism [27]. Interestingly, early studies from 1970 demonstrated that adenovirus plaque formation can be enhanced in the presence of magnesium [70], suggesting that certain ions can improve the release and spread of adenovirus. In addition, our group isolated a mutant through an in vivo bioselection strategy capable of enhancing adenovirus release through alteration of calcium homeostasis [7]. The possibility that modification of ion homeostasis could be used to promote viral egress deserves further consideration.

\section{ACKNOWLEDGEMENTS}

We thank Ramon Alemany and Manel Cascallo for valuable advice, Christopher Maxwell for extensive revision of this manuscript, and Cristina Fillat for the invitation to write this review. Research in the authors' laboratory is supported by BIO2008-04692-Co3-01 from the Ministerio de Ciencia y Tecnología of the Government of Spain (RA), EU $6^{\text {th }}$ FP research contract 18700 (Theragpox), 2005 SGR 00727 from the department d'Univesitats, Recerca i Societat de la Informació of the Generalitat de Catalunya, Mutua Madrileña Medical Research Foundation, and the Network of cooperative Research on Cancer (C03-10), Instituto de Salud Carlos III of the Ministerio de Sanidad y Consumo, Government of Spain.

\section{REFERENCES}

[1] Doronin K, Toth K, Kuppuswamy M, Ward P, Tollefson AE, Wold WS. Tumor-specific, replication-competent adenovirus vectors overexpressing the adenovirus death protein. J Virol 2000; 74: 6147-55.

[2] Sauthoff H, Heitner S, Rom WN, Hay JG. Deletion of the adenoviral E1b-19kD gene enhances tumor cell killing of a replicating adenoviral vector. Hum Gene Ther 2000; 11: 379-88.

[3] Wein LM, Wu JT, Kirn DH. Validation and analysis of a mathematical model of a replication-competent oncolytic virus for cancer treatment: implications for virus design and delivery. Cancer Res 2003; 63: 1317-24.

[4] Wodarz D. Viruses as antitumor weapons: defining conditions for tumor remission. Cancer Res 2001; 61: 3501-7.

[5] Doronin K, Kuppuswamy M, Toth K, et al. Tissue-specific, tumorselective, replication-competent adenovirus vector for cancer gene therapy. J Virol 2001; 75: 3314-24.

[6] Toth K, Djeha H, Ying B, et al. An oncolytic adenovirus vector combining enhanced cell-to-cell spreading, mediated by the ADP cytolytic protein, with selective replication in cancer cells with deregulated wnt signaling. Cancer Res 2004; 64: 3638-44.

[7] Gros A, Martinez-Quintanilla J, Puig C, et al. Bioselection of a gain of function mutation that enhances adenovirus 5 release and improves its antitumoral potency. Cancer Res 2008; 68: 8928-37.

[8] Subramanian T, Vijayalingam S, Chinnadurai G. Genetic identification of adenovirus type 5 genes that influence viral spread. J Virol 2006; 80: 2000-12.

[9] Yan W, Kitzes G, Dormishian F, et al. Developing novel oncolytic adenoviruses through bioselection. J Virol 2003; 77: 2640-50.

[10] Zhang Y, Schneider RJ. Adenovirus inhibition of cell translation facilitates release of virus particles and enhances degradation of the cytokeratin network. J Virol 1994; 68: 2544-55.
[11] Debbas M, White E. Wild-type p53 mediates apoptosis by E1A, which is inhibited by E1B. Genes Dev 1993; 7: 546-54.

[12] Chiou SK, Tseng CC, Rao L, White E. Functional complementation of the adenovirus E1B 19-kilodalton protein with Bcl-2 in the inhibition of apoptosis in infected cells. J Virol 1994; 68: 655366.

[13] Querido E, Marcellus RC, Lai A, et al. Regulation of p53 levels by the E1B 55-kilodalton protein and E4orf6 in adenovirus-infected cells. J Virol 1997; 71: 3788-98.

[14] Pilder S, Logan J, Shenk T. Deletion of the gene encoding the adenovirus 5 early region $1 \mathrm{~b} 21,000$-molecular-weight polypeptide leads to degradation of viral and host cell DNA. J Virol 1984; 52: 664-71.

[15] Tollefson AE, Scaria A, Hermiston TW, Ryerse JS, Wold LJ, Wold WS. The adenovirus death protein $(\mathrm{E} 3-11.6 \mathrm{~K})$ is required at very late stages of infection for efficient cell lysis and release of adenovirus from infected cells. J Virol 1996; 70: 2296-306.

[16] Tollefson AE, Ryerse JS, Scaria A, Hermiston TW, Wold WS. The E3-11.6-kDa adenovirus death protein (ADP) is required for efficient cell death: characterization of cells infected with adp mutants. Virology 1996; 220: 152-62.

[17] Tollefson AE, Scaria A, Saha SK, Wold WS. The 11,600-MW protein encoded by region E3 of adenovirus is expressed early but is greatly amplified at late stages of infection. J Virol 1992; 66: 3633-42.

[18] Lavoie JN, Nguyen M, Marcellus RC, Branton PE, Shore GC. E4orf4, a novel adenovirus death factor that induces p53independent apoptosis by a pathway that is not inhibited by zVADfmk. J Cell Biol 1998; 140: 637-45.

[19] Marcellus RC, Lavoie JN, Boivin D, Shore GC, Ketner G, Branton PE. The early region 4 orf 4 protein of human adenovirus type 5 induces p53-independent cell death by apoptosis. J Virol 1998; 72: 7144-53.

[20] Abou El Hassan MA, van der Meulen-Muileman I, Abbas S, Kruyt FA. Conditionally replicating adenoviruses kill tumor cells via a basic apoptotic machinery-independent mechanism that resembles necrosis-like programmed cell death. J Virol 2004; 78: 12243-51.

[21] Ito H, Aoki H, Kuhnel F, et al. Autophagic cell death of malignant glioma cells induced by a conditionally replicating adenovirus. J Natl Cancer Inst 2006; 98: 625-36.

[22] Jiang H, Gomez-Manzano C, Aoki H, et al. Examination of the therapeutic potential of Delta-24-RGD in brain tumor stem cells: role of autophagic cell death. J Natl Cancer Inst 2007; 99: 1410-4.

[23] Alonso MM, Jiang H, Yokoyama T, et al. Delta-24-RGD in combination with RAD001 induces enhanced anti-glioma effect via autophagic cell death. Mol Ther 2008; 16: 487-93.

[24] Baird SK, Aerts JL, Eddaoudi A, Lockley M, Lemoine NR, McNeish IA. Oncolytic adenoviral mutants induce a novel mode of programmed cell death in ovarian cancer. Oncogene 2008; 27: 3081-90.

[25] Ulasov IV, Tyler MA, Zhu ZB, Han Y, He TC, Lesniak MS. Oncolytic adenoviral vectors which employ the survivin promoter induce glioma oncolysis via a process of beclin-dependent autophagy. Int J Oncol 2009; 34: 729-42.

[26] Kuppuswamy M, Spencer JF, Doronin K, Tollefson AE, Wold WS, Toth K. Oncolytic adenovirus that overproduces ADP and replicates selectively in tumors due to hTERT promoter-regulated E4 gene expression. Gene Ther 2005; 12: 1608-17.

[27] Doronin K, Toth K, Kuppuswamy M, Krajcsi P, Tollefson AE, Wold WS. Overexpression of the ADP (E3-11.6K) protein increases cell lysis and spread of adenovirus. Virology 2003; 305: 378-87.

[28] Ramachandra M, Rahman A, Zou A, et al. Re-engineering adenovirus regulatory pathways to enhance oncolytic specificity and efficacy. Nat Biotechnol 2001; 19: 1035-41.

[29] Yun CO, Kim E, Koo T, Kim H, Lee YS, Kim JH. ADPoverexpressing adenovirus elicits enhanced cytopathic effect by induction of apoptosis. Cancer Gene Ther 2005; 12: 61-71.

[30] Barton KN, Paielli D, Zhang Y, et al. Second-generation replication-competent oncolytic adenovirus armed with improved suicide genes and ADP gene demonstrates greater efficacy without increased toxicity. Mol Ther 2006; 13: 347-56.

[31] Freytag SO, Stricker H, Peabody J, et al. Five-year follow-up of trial of replication-competent adenovirus-mediated suicide gene therapy for treatment of prostate cancer. Mol Ther 2007; 15: 63642. 
[32] Wang Y, Hallden G, Hill R, et al. E3 gene manipulations affect oncolytic adenovirus activity in immunocompetent tumor models. Nat Biotechnol 2003; 21: 1328-35.

[33] Bortolanza S, Bunuales M, Alzuguren P, et al. Deletion of the E3$6.7 \mathrm{~K} / \mathrm{gp} 19 \mathrm{~K}$ region reduces the persistence of wild-type adenovirus in a permissive tumor model in Syrian hamsters. Cancer Gene Ther 2009.

[34] Bhat BM, Brady HA, Wold WS. Virus deletion mutants that affect a 3' splice site in the E3 transcription unit of adenovirus 2. Mol Cell Biol 1985; 5: 2405-13.

[35] Scaria A, Wold WS. Fine-mapping of sequences that suppress splicing in the E3 complex transcription unit of adenovirus. Virology 1994; 205: 406-16.

[36] Tollefson AE, Scaria A, Ying B, Wold WS. Mutations within the ADP (E3-11.6K) protein alter processing and localization of ADP and the kinetics of cell lysis of adenovirus-infected cells. J Virol 2003; 77: 7764-78.

[37] Harrison D, Sauthoff H, Heitner S, Jagirdar J, Rom WN, Hay JG. Wild-type adenovirus decreases tumor xenograft growth, but despite viral persistence complete tumor responses are rarely achieved--deletion of the viral E1b-19-kD gene increases the viral oncolytic effect. Hum Gene Ther 2001; 12: 1323-32.

[38] Liu TC, Hallden G, Wang Y et al. An E1B-19 kDa gene deletion mutant adenovirus demonstrates tumor necrosis factor-enhanced cancer selectivity and enhanced oncolytic potency. Mol Ther 2004; 9: 786-803.

[39] Liu TC, Wang Y, Hallden G, et al. Functional interactions of antiapoptotic proteins and tumor necrosis factor in the context of a replication-competent adenovirus. Gene Ther 2005; 12: 1333-46.

[40] Mi J, Li ZY, Ni S, Steinwaerder D, Lieber A. Induced apoptosis supports spread of adenovirus vectors in tumors. Hum Gene Ther 2001; 12: 1343-52.

[41] Liu TJ, Zhang WW, Taylor DL, Roth JA, Goepfert H, Clayman GL. Growth suppression of human head and neck cancer cells by the introduction of a wild-type p53 gene via a recombinant adenovirus. Cancer Res 1994; 54: 3662-7.

[42] Mujoo K, Maneval DC, Anderson SC, Gutterman JU. Adenoviralmediated p53 tumor suppressor gene therapy of human ovarian carcinoma. Oncogene 1996; 12: 1617-23.

[43] Nielsen LL, Dell J, Maxwell E, Armstrong L, Maneval D, Catino JJ. Efficacy of p53 adenovirus-mediated gene therapy against human breast cancer xenografts. Cancer Gene Ther 1997; 4: 12938.

[44] Zhang WW, Fang X, Mazur W, French BA, Georges RN, Roth JA. High-efficiency gene transfer and high-level expression of wildtype p53 in human lung cancer cells mediated by recombinant adenovirus. Cancer Gene Ther 1994; 1: 5-13.

[45] Liu Q, Gazitt Y. Adenovirus-mediated delivery of p53 results in substantial apoptosis to myeloma cells and is not cytotoxic to flowsorted $\mathrm{CD} 34(+)$ hematopoietic progenitor cells and normal lymphocytes. Exp Hematol 2000; 28: 1354-62.

[46] Zhang WW, Alemany R, Wang J, Koch PE, Ordonez NG, Roth JA. Safety evaluation of Ad5CMV-p53 in vitro and in vivo. Hum Gene Ther 1995; 6: 155-64.

[47] Hall AR, Dix BR, O'Carroll SJ, Braithwaite AW. p53-dependent cell death/apoptosis is required for a productive adenovirus infection. Nat Med 1998; 4: 1068-72.

[48] Dix BR, O'Carroll SJ, Myers CJ, Edwards SJ, Braithwaite AW. Efficient induction of cell death by adenoviruses requires binding of E1B55k and p53. Cancer Res 2000; 60: 2666-72.

[49] Royds JA, Hibma M, Dix BR, et al. p53 promotes adenoviral replication and increases late viral gene expression. Oncogene 2006; 25: 1509-20.

[50] Sauthoff H, Pipiya T, Heitner S, et al. Late expression of p53 from a replicating adenovirus improves tumor cell killing and is more tumor cell specific than expression of the adenoviral death protein. Hum Gene Ther 2002; 13: 1859-71.

[51] van Beusechem VW, van den Doel PB, Grill J, Pinedo HM, Gerritsen WR. Conditionally replicative adenovirus expressing p53 exhibits enhanced oncolytic potency. Cancer Res 2002; 62: 616571.

[52] Geoerger B, Vassal G, Opolon P, et al. Oncolytic activity of p53expressing conditionally replicative adenovirus AdDelta24-p53 against human malignant glioma. Cancer Res 2004; 64: 5753-9.

[53] Geoerger B, van Beusechem VW, Opolon P, et al. Expression of p53, or targeting towards EGFR, enhances the oncolytic potency of conditionally replicative adenovirus against neuroblastoma. J Gene Med 2005; 7: 584-94.

[54] Almasan A, Ashkenazi A. Apo2L/TRAIL: apoptosis signaling, biology, and potential for cancer therapy. Cytokine Growth Factor Rev 2003; 14: 337-48.

[55] Walczak H, Miller RE, Ariail K, et al. Tumoricidal activity of tumor necrosis factor-related apoptosis-inducing ligand in vivo. Nat Med 1999; 5: 157-63.

[56] Ashkenazi A, Pai RC, Fong S, et al. Safety and antitumor activity of recombinant soluble Apo2 ligand. J Clin Invest 1999; 104: 15562.

[57] Sova P, Ren XW, Ni S, et al. A tumor-targeted and conditionally replicating oncolytic adenovirus vector expressing TRAIL for treatment of liver metastases. Mol Ther 2004; 9: 496-509.

[58] Wohlfahrt ME, Beard BC, Lieber A, Kiem HP. A capsid-modified, conditionally replicating oncolytic adenovirus vector expressing TRAIL Leads to enhanced cancer cell killing in human glioblastoma models. Cancer Res 2007; 67: 8783-90.

[59] Qiu S, Ruan H, Pei Z, et al. Combination of Targeting GeneViroTherapy with 5-FU enhances antitumor efficacy in malignant colorectal carcinoma. J Interferon Cytokine Res 2004; 24: 219-30.

[60] Pan Q, Liu B, Liu J, Cai R, Wang Y, Qian C. Synergistic induction of tumor cell death by combining cisplatin with an oncolytic adenovirus carrying TRAIL. Mol Cell Biochem 2007; 304: 315-23.

[61] Alemany R, Cascallo M. Oncolytic viruses from the perspective of the immune system. Future Microbiol 2009; 4: 527-36.

[62] Aghi M, Martuza RL. Oncolytic viral therapies - the clinical experience. Oncogene 2005; 24: 7802-16.

[63] Thomas MA, Spencer JF, Toth K, Sagartz JE, Phillips NJ, Wold WS. Immunosuppression enhances oncolytic adenovirus replication and antitumor efficacy in the Syrian hamster model. Mol Ther 2008; 16: 1665-73

[64] Ganesh S, Gonzalez-Edick M, Gibbons D, Van Roey M, Jooss K. Intratumoral coadministration of hyaluronidase enzyme and oncolytic adenoviruses enhances virus potency in metastatic tumor models. Clin Cancer Res 2008; 14: 3933-41.

[65] Ganesh S, Gonzalez Edick M, Idamakanti N, et al. Relaxinexpressing, fiber chimeric oncolytic adenovirus prolongs survival of tumor-bearing mice. Cancer Res 2007; 67: 4399-407.

[66] Kim JH, Lee YS, Kim H, Huang JH, Yoon AR, Yun CO. Relaxin expression from tumor-targeting adenoviruses and its intratumoral spread, apoptosis induction, and efficacy. J Natl Cancer Inst 2006; 98: 1482-93.

[67] van Kuppeveld FJ, Hoenderop JG, Smeets RL, et al. Coxsackievirus protein $2 \mathrm{~B}$ modifies endoplasmic reticulum membrane and plasma membrane permeability and facilitates virus release. Embo J 1997; 16: 3519-32.

[68] Agirre A, Barco A, Carrasco L, Nieva JL. Viroporin-mediated membrane permeabilization. Pore formation by nonstructural poliovirus 2B protein. J Biol Chem 2002; 277: 40434-41.

[69] Gonzalez ME, Carrasco L. Viroporins. FEBS Lett 2003; 552: 28 34.

[70] Williams JF. Enhancement of adenovirus plaque formation on HeLa cells by magnesium chloride. J Gen Virol 1970; 9: 251-5.

(C) Gros and Guedan.; Licensee Bentham Open

This is an open access article licensed under the terms of the Creative Commons Attribution Non-Commercial License (http://creativecommons.org/licenses/by-nc/3.0/) which permits unrestricted, non-commercial use, distribution and reproduction in any medium, provided the work is properly cited. 